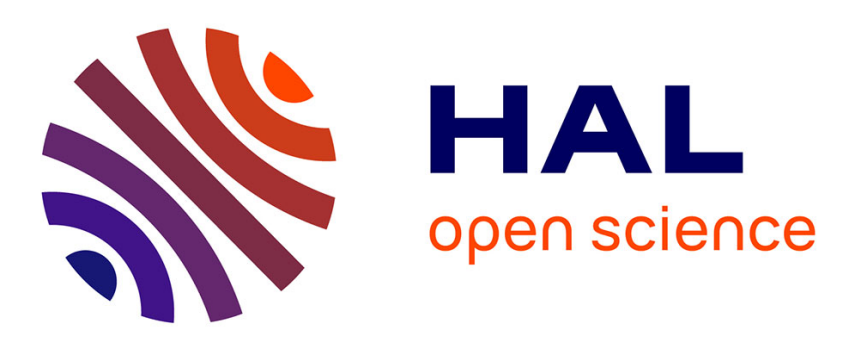

\title{
Fuel cell remaining useful life prediction and uncertainty quantification under an automotive profile
}

Mathieu Bressel, Mickaël Hilairet, Daniel Hissel, Belkacem Bouamama

\section{To cite this version:}

Mathieu Bressel, Mickaël Hilairet, Daniel Hissel, Belkacem Bouamama. Fuel cell remaining useful life prediction and uncertainty quantification under an automotive profile. Annual Conference of the IEEE Industrial Electronics Society, Oct 2016, Firenze (Florence), Italy. hal-02380405

\section{HAL Id: hal-02380405 \\ https://hal.science/hal-02380405}

Submitted on 26 Nov 2019

HAL is a multi-disciplinary open access archive for the deposit and dissemination of scientific research documents, whether they are published or not. The documents may come from teaching and research institutions in France or abroad, or from public or private research centers.
L'archive ouverte pluridisciplinaire HAL, est destinée au dépôt et à la diffusion de documents scientifiques de niveau recherche, publiés ou non, émanant des établissements d'enseignement et de recherche français ou étrangers, des laboratoires publics ou privés. 


\title{
Fuel Cell Remaining Useful Life Prediction and Uncertainty Quantification Under an Automotive Profile
}

\author{
Mathieu Bressel $^{* \dagger}$, Mickael Hilairet*, Daniel Hissel ${ }^{*}$, and Belkacem Ould Bouamama ${ }^{\dagger}$ \\ * Franche-Comté Electronique Mécanique Thermique et Optique - Sciences et Technologies (FEMTO-ST), \\ UMR CNRS 6174, FCLAB, FR CNRS 3539, University of Bourgogne Franche-Comté, Belfort, France \\ ${ }^{\dagger}$ CRIStAL, UMR CNRS 9189, Université Sciences et Technologies, Villeneuve d’ Ascq, France
}

\begin{abstract}
A Proton exchange membrane fuel cell is a clean and efficient energy converter that can be use to power an electrical vehicle efficiently. Nevertheless, degradation mechanisms affect the lifespan of this electrochemical converter. Consequently, the estimation of the State of Health and Remaining Useful Life have been the subject of numerous researches in the past years. However, most of the methods available in the literature dealing with fuel cell prognostic do not allow the uncertainty quantification of the estimation that can be implemented online due to the computational cost. As a novelty, this paper presents a prognostic algorithm based on an Extended Kalman Filter. This observer estimates the State of Health, the speed of the degradation and also provides the estimation uncertainty. Then, an Inverse First Order Reliability Method computes the Remaining Useful Life with a $90 \%$ confidence interval based on the estimation of the observer. This method is applied on a 175 hours dataset coming from a experimental test on a 8-cells fuel cell stack subjected to an automotive power profile.
\end{abstract}

Index Terms-Remaining Useful Life, PEM Fuel Cell, Extended Kalman Filter, Uncertainty Quantification

\section{INTRODUCTION}

Since fossil energy assets are decreasing, a transition to renewable energy is required. One of the significant issue is the capacity to store the produced electricity which can be handled by the utilization of hydrogen as an energy vector which delivers power through a fuel cell [1]. Those electrochemical converters, including the Proton Exchange Membrane Fuel Cell (PEMFC), receive a developing enthusiasm from scientific and industrial groups around the world. PEMFC is a promising substitute for internal combustion engine for efficient and clean transportation applications, additionally in a bigger scale, fuel cells are able to produce heat and electricity in a combined manner for a whole building ( $\mu$-CHP) [2]. Notwithstanding, those promising converters experience a restricted lifetime due to not completely understood electrochemical degradation that avoid an industrial deployment [3]. Additionally, the operating condition and load profile affects the degradation speed which makes the prediction of the Remaining Useful Life (RUL) challenging [4].

This is the motivation behind why Prognostics and Health Management (PHM) of PEMFC is gaining awareness in the research field. It permits the extension of the life of this electrochemical converter due to monitoring, diagnosis, prognostic, and corrective actions at a decision level [5][7]. By selecting particular features from the data, one can build indicators of the State of Heath $(\mathrm{SoH})$ and track their evolutions with the aim of predicting the End of Life (EoL).

The prognostic activity focuses on the evaluation of the $\mathrm{SoH}$ and on the prediction of the upcoming behavior, yet it should likewise have the capability to estimate the confidence of the prediction [8]. The certainty of RUL prediction can be assessed by analytical methods, for instance, the First Order Reliability Method (FORM) which can be implemented in real time due to a low computational cost [9]. Regardless of the numerous application of RUL prediction of PEMFC that can be found in the literature, three papers only addresses the uncertainty quantification issue [10]-[12].

As a novelty, the presented work contributes to model-based prognostics of PEMFC subjected to an automotive current profile. It aims at building a robust algorithm able to predict the RUL in real time and to assess the confidence using an observer and the Inverse First Order Reliability Method (IFORM).

Section 2 describes briefly the test bench and the performed test. The methodology for $\mathrm{SoH}$ estimation and uncertainty quantification using an Extended Kalman Filter (EKF) is presented in Section 3. It also describes the IFORM which is applied to a 8-cells stack under an automotive profile. A conclusion and some perspectives are given in the last section.

\section{EXPERIMENTAL SETUP}

To observe the aging, a 175 hours continuous experimental test is performed on an 8-cells fuel cell stack with a surface of $220 \mathrm{~cm}^{2}$ provided by the French Atomic Energy and Alternative Energies Commission (CEA). A 10kW test bench (Fig. 1) regulates the temperature by mean of a cooling system while the stoichiometry and pressure are controlled continuously. Moreover, the test bench supplies the fuel cell in humidified hydrogen and air at the anode and cathode respectively. Tab. I shows the operating conditions.

During the long time test, the PEMFC is subjected to an automotive profile of current while the stack voltage is recorded with an hourly sample time as seen on Fig. 2. It aims at simulating the power demand of an electrical vehicle 


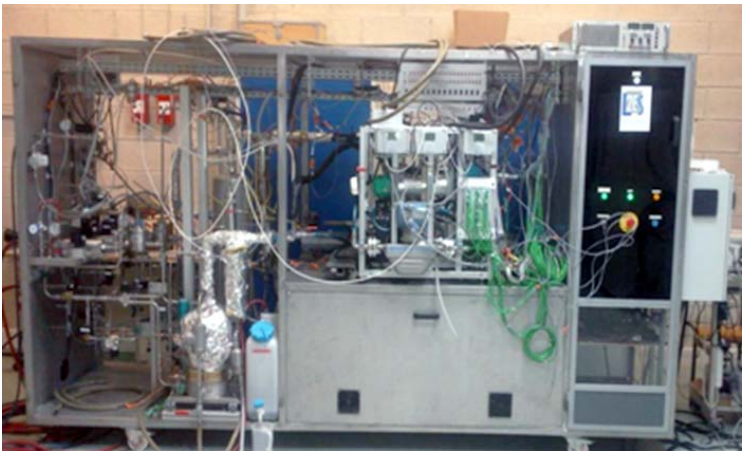

Fig. 1. 10kW in-lab test bench

TABLE I

OPERATING CONDITIONS

\begin{tabular}{|c|c|}
\hline Parameter & Value \\
\hline Temperature & $80^{\circ} \mathrm{C}$ \\
Anode and cathode stoichiometry ratios & $1.5-2$ \\
Absolute pressure anode/cathode & $1.5 \mathrm{bar}$ \\
Relative humidity anode/cathode & $50 \%$ \\
Nominal current density $i_{\text {nom }}$ & $0.45 \mathrm{~A} . \mathrm{cm}^{-2}$ \\
Maximal current density $i_{\max }$ & $0.77 \mathrm{~A} \cdot \mathrm{cm}^{-2}$ \\
\hline
\end{tabular}

and follow the cycles (as seen in the zoom of the voltage on Fig. 2):

- A current density corresponding to a cell voltage of $0,9 \mathrm{~V}$ during 10s.

- A current density corresponding to a cell voltage of $0,7 \mathrm{~V}$ during 50s.

In addition, periodically during the test, static responses of the PEMFC is measured with polarization curves (as shown in Fig. 3). After each of those characterization, the level of current is adjusted is order to complete the defined mission.

\section{Prognostics of PEM Fuel Cell}

\section{A. Generalities and method}

The prognostic activity is defined as "the prediction of the remaining time before one or more failure modes appear
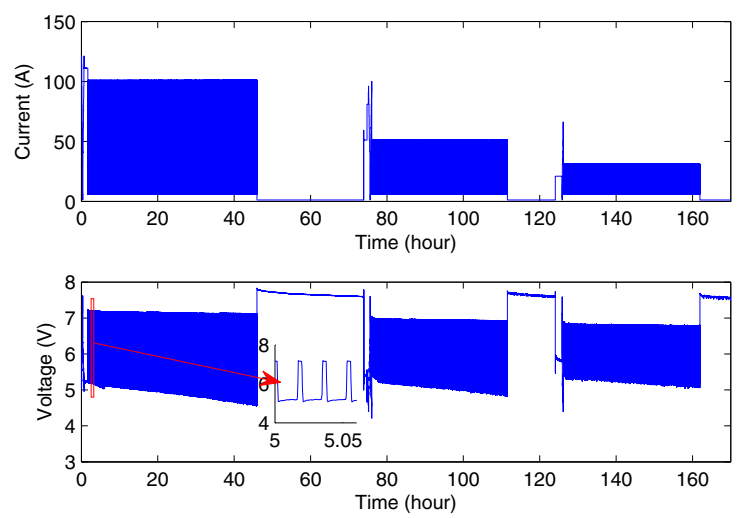

Fig. 2. Load current and recorded stack voltage

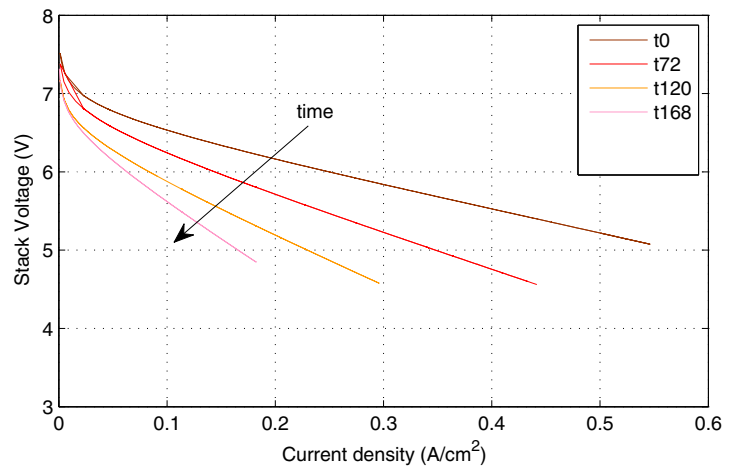

Fig. 3. Polarization curves during the ageing

avoiding a system to fulfill a given mission" [13]. It is therefore carried out in two steps:

- first, the current $\mathrm{SoH}$ is estimated

- then, the damage progression is forecasted until a threshold is reached at the EoL

In other words, the RUL is defined as the time difference between the predicted EoL $t_{E o L}$ and the time the prediction is made $t_{k}$ as:

$$
R U L\left(t_{k}\right)=t_{E o L}-t_{k}
$$

Numerous model-based methods for RUL prediction have been developed. For each, an analytical relation is used to express the degradation model which can have a physical meaning when expert knowledge is available. Nevertheless, as stated earlier, it is challenging to model the several degradation mechanisms that happen within the fuel cell, it is therefore chosen to develop an empirical relation in this work.

Model-based method which have been successfully applied to PEMFC RUL estimation, was never applied to an automotive dataset where the dynamics are fast. In addition, the uncertainty quantification issue is adressed in [11] by mean of a Particle Filter which is challenging to implement online [14]. As an extension of a previous work [12], the method presented in this paper (Fig. 4) allows the estimation of the SoH and RUL of a PEMFC under a fast dynamical load profile with uncertainty quantification. It consists in several steps:

1) During the long term test, characterizations are performed (e.g. the polarization curve of Fig. 3).

2) From those, a nonlinear optimization algorithm is applied to fit a model in order to extract the electrochemical parameters of the PEMFC. The pertinent parameters that evolve the most will be selected.

3) An empirical model of parametric degradation is then built (see Section 3.B).

4) An observer then estimates the $\mathrm{SoH}$ and the confidence in real time (see Section 3.C).

5) When considered as random variables, the $\mathrm{SoH}$ and the speed of degradation allows the IFORM to predict the RUL with probability bounds (see Section 3.D).

Usually, the confidence in the state estimation of the EKF has been extensively used for assessing the precision [15]. 
Real Fuel Cell

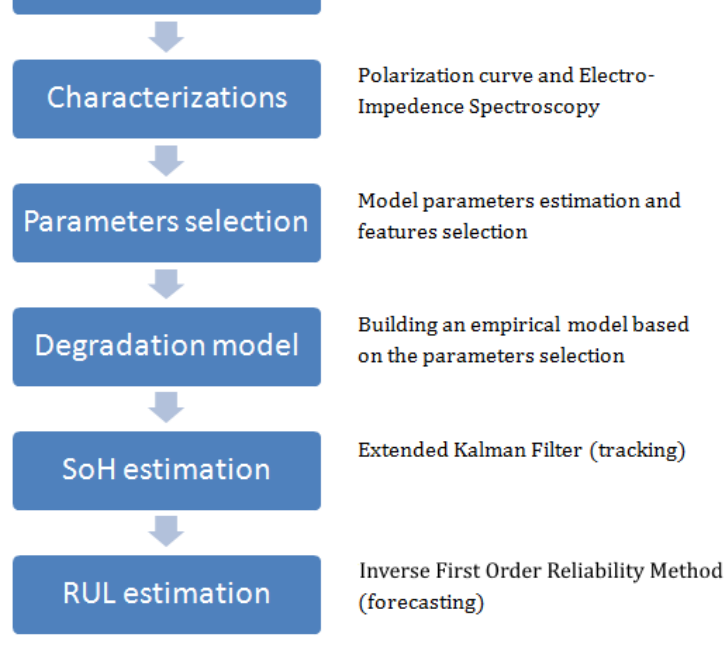

Fig. 4. Model-based prognostics methodology for PEMFC

Despite the fact that the value of the computed uncertainty is strongly dependent on the setting of the observer, it is usually not discussed in the consulted literature.

In this paper, the algorithm presents the advantages of handling a change in temperature, load and work for several PEMFC under the condition of having the initial polarization curve. The global method does not require a lot of data to estimate the RUL with a high certainty compared to data-based methods. Moreover, unlike hybrid and data based methods, this work can be implemented online as discussed in the section 3.E.

\section{B. Design of the degradation model}

As described in the second step of the method, a LevenbergMarquardt optimization algorithm extracts some electrochemical parameters of the following equation, well known for describing the static behavior of a PEMFC, on every polarization curve (see Fig. 3) :

$$
V_{s t}=n \cdot\left(E_{0}-A \cdot T \cdot \ln \left(\frac{i}{i_{0}}\right)-R \cdot i-B \cdot T \cdot \ln \left(1-\frac{i}{i_{L}}\right)\right)
$$

where $V_{s t}$ is the stack voltage, $n$ is the number of cells of the stack, $i$ is the load current, $T$ is the stack temperature. $E_{0}$ is the maximum voltage the fuel cell is able to reach. This voltage is lowered by several losses namely: ohmic losses (R.i), activation losses due to the kinetic of the chemical reaction (A.T. $\ln \left(\frac{i}{i_{0}}\right)$ ) and concentration losses (representing the effect of a local lack of reactant at high currents) [16]. The equation above being of a nonlinear nature, the optimization algorithm needs to be initiated with standard value found in the literature in order to reach the true value of the parameters [16]. The extracted parameters of Equ. 2 are:

- The Open Circuit Voltage (OCV) $E_{0}$

- The exchange current $i_{0}$
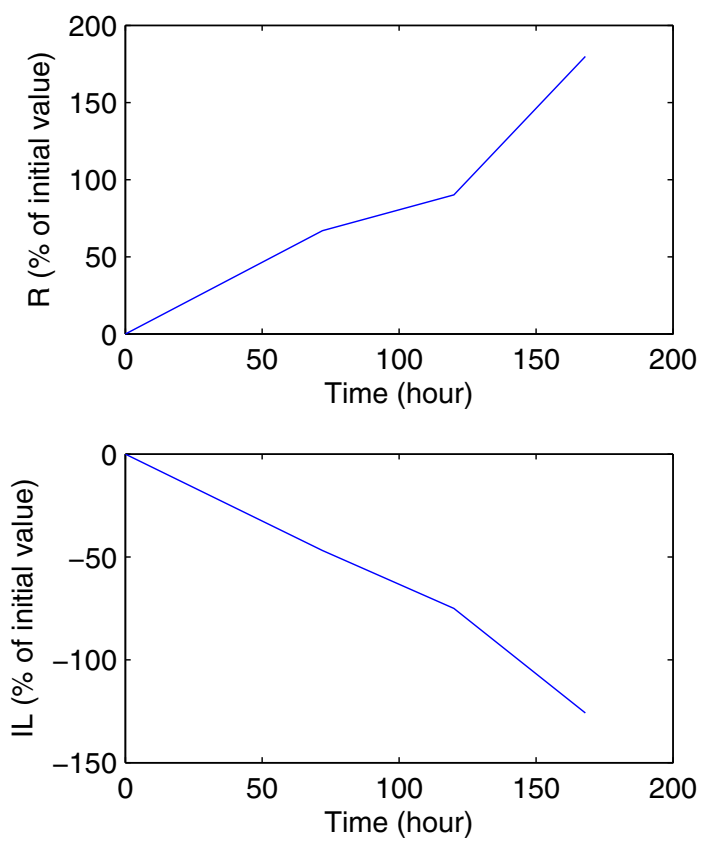

Fig. 5. Evolution of the selected electro-chemical parameters through aging

- The global resistance $R$ (membranes, connectors, end plates, etc.)

- The limiting current $i_{L}$

Among them, only $R$ and $i_{L}$ shows a significant evolution as shown in Fig. 5. Some physical interpretation of those degradations can be found in a previous work [12].

Once that it is not possible to link the speed of degradation and the operating conditions using a physical law, the parameters evolution is modeled with a linear equation. Furthermore, the loss caused by the limit current and the one caused by the resisitivity from Equ. 2 can not be seperated. It is therefore chosen to couple the deviation of those parameters with the SoH variable $\alpha(t)$ leading to the following degradation model:

$$
\begin{gathered}
R(t)=R_{0}(1+\alpha(t)), I_{L}(t)=I_{L 0}(1-\alpha(t)), \\
\alpha(t)=\int_{0}^{t} \beta(t) d t
\end{gathered}
$$

where $\beta$ is the speed of degradation.

\section{Observer-based $\mathrm{SoH}$ estimation}

1) Problem formulation: The joint estimation of the PEMFC degradation indicator $\alpha_{k}$ and of the speed of degradation $\beta_{k}$ is based on the discrete nonlinear system:

$$
\begin{gathered}
x_{k+1}=A x_{k}+w_{k} \\
y_{k}=g\left(x_{k}, u_{k}\right)+v_{k}
\end{gathered}
$$

with $x_{k}=\left[\alpha_{k} \beta_{k}\right]^{T}$ the state of the system, $u_{k}$ the inputs (current, temperature), $y_{k}$ is the output voltage, $w_{k}$ and $v_{k}$ are process and observation noises supposed Gaussian with zero mean and of variances $\mathrm{Q}$ and $\mathrm{R}$ respectively. $g\left(x_{k}, u_{k}\right)$ 
is derived from Equ.2 in discrete form and expressed with regard to $\alpha_{k}$. In this state and parameter estimation problem, the transition matrix is expressed:

$$
A=\left[\begin{array}{cc}
1 & T_{S} \\
0 & 1
\end{array}\right]
$$

where $T_{S}$ is the sampling time of the regular discrete Extended Kalman Filter which is expressed as:

$$
\begin{aligned}
& \text { Initialization } \\
& x_{0 \mid 0}=E\left[x\left(t_{0}\right)\right] \\
& P_{0 \mid 0}=\operatorname{Var}\left[x\left(t_{0}\right)\right]
\end{aligned}
$$

\section{Prediction}

$$
\begin{aligned}
& x_{k \mid k-1}=A x_{k-1 \mid k-1} \\
& P_{k \mid k-1}=A P_{k-1 \mid k-1} A^{T}+Q
\end{aligned}
$$

\section{Correction}

$$
\begin{aligned}
& K_{k}=P_{k \mid k-1} H_{k}^{T}\left(H_{k} P_{k \mid k-1} H_{k}^{T}+R\right)^{-1} \\
& \text { with } H_{k}=\frac{\partial g\left(x_{k}, u_{k}\right)}{\partial x_{k}} \\
& P_{k \mid k}=\left(I-K_{k} H_{k}\right) P_{k \mid k-1} \\
& x_{k \mid k}=x_{k \mid k-1}+K_{k}\left(V_{s t k}-g\left(x_{k}, u_{k}\right)\right)
\end{aligned}
$$

The choice of the EKF is motivated due to the fact that the algorithm allows to estimate the $\mathrm{SoH}$ and the speed of degradation for different conditions of current and temperature. In addition, through the covariance matrix of the estimates error $P_{k \mid k}$, the EKF provides the uncertainty of the state estimation for a correct setting of $Q$ and $R$ as discussed in the following section.

2) Setting and uncertainty of the state estimation: To initialize the observer, the initial state vector is set to zero since the speed of degradation is assumed unknown, $x_{0 \mid 0}=\left[\begin{array}{ll}0 & 0\end{array}\right]^{T}$. Moreover, the value of the initial covariance matrix of the error of estimation $P_{0 \mid 0}$ is obtained by solving the algebraic Riccati equation in steady state when $P_{k \mid k}=P_{k-1 \mid k-1}$ :

$$
A P_{k \mid k} A^{T}-P_{k \mid k}-A P_{k \mid k} H_{k}^{T}\left(H_{k} P_{k \mid k} H_{k}^{T}+R\right)^{-1} H_{k} P_{k \mid k} A^{T}+Q=0
$$

with $H_{k}$ the observation matrix for the initial conditions of current, temperature and state.

The EKF gives the optimal state estimation $x_{k}^{\star}$ given by the conditional probability density function:

$$
p\left(x_{k} \mid y_{k}\right) \sim N\left(x_{k \mid k}, P_{k \mid k}\right)
$$

with $x_{k \mid k}$ the expected value and $P_{k \mid k}$ defined as:

$$
P_{k \mid k}=\left[\begin{array}{cc}
\sigma_{\alpha_{k}}^{2} & 0 \\
0 & \sigma_{\beta_{k}}^{2}
\end{array}\right]
$$

The value of $P_{k \mid k}$ is dependent on the process and measure noise variances in the Kalman theory. Nevertheless, $Q$ and $R$ are usually considered as tuning variables. Therefore, the true uncertainty of the state estimation can be obtained only with a proper setting of $Q$ and $R$. By computing the square of the standard deviation on the measured voltage (see Fig. 2), one can obtain the value of the measurement noise variance. If the EKF runs at a different sampling rate than the recorded voltage by the test bench $T_{s_{\text {bench }}}$, the discrete variance of the measurement noise can be adapted using:

$$
R_{T_{s}} \cong \frac{\sigma^{2} \cdot T_{s}}{T_{s_{\text {bench }}}}
$$

It is not trivial to obtain analytically the process noise variance value. It is chosen to minimize a quadratic cost function on a constant load aging dataset performed on the same test bench as seen in Equ.13. This function is also multiplied by the sample number once that the algorithm should give more accurate estimations in time. The diagonal term $Q_{11}$ is set to zero, once that $\alpha_{k}$ is the integral of $\beta_{k}$ in the model.

$$
\begin{aligned}
Q & =\left[\begin{array}{cc}
Q_{11} & 0 \\
0 & Q_{22}
\end{array}\right] \\
J\left(Q_{22}\right) & \left.=\frac{1}{n} \sum_{k=0}^{n-1}\left(\alpha_{k \mid k}-\alpha_{k}\right)\right)^{2} \cdot k
\end{aligned}
$$

where $\alpha_{k \mid k}$ is the estimation of the SoH at sample $k, \alpha_{k}$ is the real $\mathrm{SoH}$ at sample $k$, and $n$ is the total number of samples. Similarly to Equ.11, the discrete variance of the process noise should be adapted if the EKF runs at a different sampling rate as:

$$
Q_{T_{s}} \cong \frac{Q_{22} \cdot T_{s}}{T_{s_{\text {bench }}}}
$$

Once that the EKF is correctly set, the uncertainty on the $\mathrm{SoH}$ and speed of degradation estimations are given by the diagonal terms of the covariance matrix $P_{k \mid k}$.

\section{IFORM for RUL estimation}

1) IFORM algorithm: The quantification of the uncertainty in the SoH or RUL is usually based on Monte Carlo simulation and is therefore computationally expensive. To implement a fast confidence algorithm, one can use analytical method such as the IFORM which can be used for the estimation of an unknown parameter for a given failure probability level [17]. In the IFORM, the limit between healthy and failure state is represented with the limit state function $g(u, y)$ (see Fig. 6) where $u$ is the vector of random state variable $x_{k \mid k}=\left[\alpha_{k \mid k}, \beta_{k \mid k}\right]^{T}$ expressed in the standard normalized space and $y$ is the number of clock. In this work, the fuel cell is

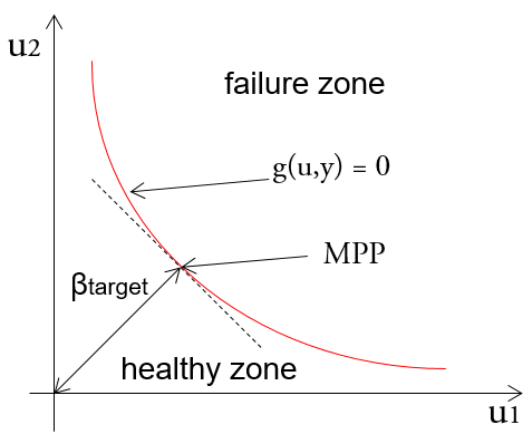

Fig. 6. Limit state function and Most Probable Point 
considered in the failure zone when the estimated $\mathrm{SoH}$ crosses a threshold: $\alpha_{k \mid k} \geq \alpha_{\max }$. Consequently, the limit state function is expressed:

$$
g(u, y)=R U L_{k}(u)-y
$$

where the predicted RUL depends on the $\mathrm{SoH}$ and speed of degradation at sample $k$ as [18]:

$$
R U L_{k}=\frac{\alpha_{\max }-\alpha_{k \mid k}}{\beta_{k \mid k} \cdot T_{s}}
$$

The IFORM algorithm aims at finding the Most Probable Point (MPP) in the standard normalized space using a gradient descent technique satisfying constraints for a given failure probability level $P_{f}$. For a given $P_{f}$ corresponds a reliability index $\beta_{\text {target }}$ (not to confuse with the speed of degradation $\beta_{k \mid k}$ ) which is calculated using the Inverse Cumulative Distribution Function (I-CDF) of the failure probability $P_{f}$ as:

$$
\beta_{\text {target }}=\Phi^{-1}\left(P_{f}\right)
$$

The reliability index $\beta_{\text {target }}$ is defined as the minimal distance between the origin and the limit state function in the normalized space, corresponding to the coordinates of the MPP:

$$
\|u\|=\beta_{\text {target }}
$$

The IFORM follows the iterative procedure:

1) The counter $\mathrm{j}$ is set to zero and an initial guess for the MPP is chosen $x^{j}=\left\{x_{1}^{j}, x_{2}^{j}\right\}$

2) The coordinates are transformed into normal space using the mean and variance from the $\operatorname{EKF}\left(\mu_{i}\right.$ and $\sigma_{i}$ respectively):

$$
u_{i}^{j}=\frac{x_{i}^{j}-\mu_{i}}{\sigma_{i}}
$$

3) The gradient vector of the limit state function is computed:

$$
a^{j}=\frac{\partial g}{\partial u^{j}}=\left[\begin{array}{c}
\frac{-1}{T_{s} u_{2}^{j}} \\
\frac{-\left(\alpha_{n o r m m a x}-u_{1}^{j}\right)}{T_{s}\left(u_{2}^{j}\right)^{2}}
\end{array}\right]
$$

4) The next point is computed using:

$$
u^{j+1}=-\frac{a^{j}}{\left|a^{j}\right|} \cdot \beta_{\text {target }}
$$

5) $x^{j+1}$ is computed by transforming back into the original space and the steps are repeated from 3 until the algorithm converges (usually in 4-5 iterations).

The convergence is insured when two criteria are satisfied (using tolerance $\delta_{1}$ and $\delta_{2}$ ):

- The MPP lies in the limit state function:

$$
\left|g\left(x^{j}\right)-y\right| \leq \delta_{1}
$$

- The coordinates of the point are nearly constant between two iterations:

$$
\left|x^{j+1}-x^{j}\right| \leq \delta_{2}
$$

By repeating this method for several failure probability level $P_{f}$ (so for several $\beta_{\text {target }}$ ), one can compute probability bounds
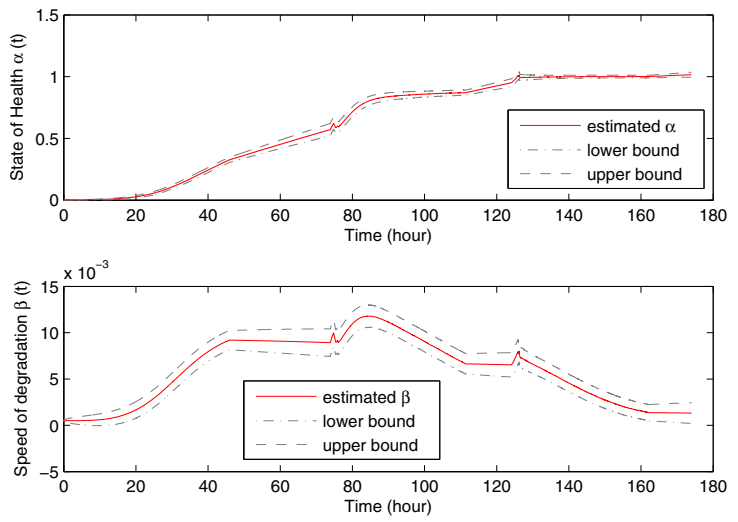

Fig. 7. $\alpha_{k}$ and $\beta_{k}$ estimation with the $99 \%$ confidence interval

(for instance, the RUL with a confidence of $90 \%$ is given by $P_{f}=\{0.05,0.5,0.95\}$ which means $\beta_{\text {target }}=\{-1.7,0,1.7\}$ ) [19].

\section{E. Experimental results and discussion}

1) Results: The methodology presented above is applied to the dataset of Section 2. First, the fuel cell model parameters of Equ. $2\left\{E_{0}, I_{0}, R_{0}, I_{L 0}\right\}$ are obtained using a polarization curve and the Levenberg-Marquardt optimization method of section 3.B. Secondly, with the pair current-voltage as an input (see Fig. 2), the EKF estimates the SoH $\alpha_{k \mid k}$ and the speed of degradation $\beta_{k \mid k}$ with a $99 \%$ confidence interval $(3 \sigma)$ at each second of the $175 \mathrm{~h}$ dataset as seen in Fig. 7. Finally, using the estimation of the EKF, the IFORM predicts the RUL with a confidence of $90 \%$ (see Fig. 8).

In order to assess the accuracy of the results, several performance metrics are used. Once that the true value of $\{\alpha(t), \beta(t)\}$ can not be obtained in-lab, the performance evaluation of the state estimation can not be performed. However, the RMSE of the stack voltage allows to assess the good estimation of the observer which is of about $10 \%$. This estimation accuracy can be reached only if the state is well estimated. From Fig. 7, one can notice that the current profile affects the speed of degradation in such a way that the RUL is re-evaluated at each change in the load current value.

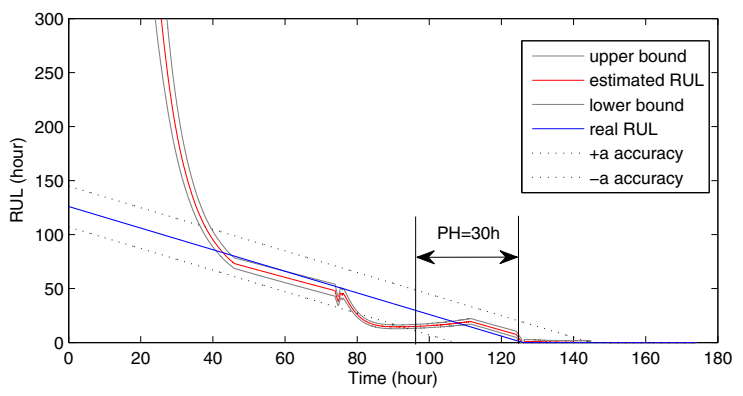

Fig. 8. RUL estimation with $90 \%$ probability bounds and $\mathrm{PH}$ metric 
The chosen threshold for EoL of the PEMFC is a maximum parameter deviation $\alpha_{\max }=100 \%$ which seems to be reached from $t=124 \mathrm{~h}$. The accuracy assessment of the RUL prediction is accomplished with the Prognostic Horizon metric (PH). It asses the time for which the RUL prediction is within defined bounds till the end of life. The $\mathrm{PH}$ (with $\mathrm{a}=0.15$ ) is equal to 30 hours as seen on Fig. 8. The uncertainty is bounded by the PH metric at $t=96$ hours. The global method is able to estimate the RUL with a high confidence after 40 hours which is suited for an automotive application. Moreover, the algorithm only take $22 \mathrm{~s}$ to compute the $175 \mathrm{~h}$ of data (with a sampling period of 1 sample/s) on MATLAB-Simulink using an Intel i5 Processor, $2.40-\mathrm{GHz}$ clock frequency, and 4-GB RAM which is promising for an online implementation of the algorithm. Nevertheless, between $t=80 \mathrm{~h}$ and $t=100 \mathrm{~h}$, the RUL is underestimated due to a higher speed of degradation. This issue could be avoided with a model linking the speed of degradation and the operating conditions.

2) Discussion: The algorithm presented in this work gives promising results despite an unknown variable speed of degradation in an extremely dynamical current profile. Furthermore, the method is able to provide the confidence in the $\mathrm{SoH}$ estimation and RUL prediction with a low computational cost thanks to the setting of the covariance of the process and measurement noises. However, the measurement noise is not constant in practice and so the confidence interval could be enhanced with an online standard deviation estimation of this noise [20].

\section{CONCLUSION}

A model-based method for PEMFC prognostics is presented. It allows the estimation of the $\mathrm{SoH}$, speed of degradation and RUL with confidence intervals. In order to predict the aging of the fuel cell, an Extended Kalman Filter use an empirical model of degradation build after a parameter analysis. This analysis is performed thanks to a Levenberg-Marquardt optimization algorithm which allows to obtain the evolution of the parameters. The issue of uncertainty quantification of the state estimation is also addressed by mean of the setting of the EKF. Finally, the IFORM allows the RUL prediction with a low computational cost, a high accuracy and the confidence in the prediction is given as well. This method is applied to a 8-cells PEMFC under an automotive current profile, and it is able to give auspicious results even in the presence of an unknown speed of degradation on a highly dynamical profile.

\section{ACKNOWLEDGMENT}

This work was supported by the project ANR PROPICE (ANR-12-PRGE-0001) and by the project Labex ACTION (ANR-11-LABX-01-0) both funded by the French National Research Agency.

\section{REFERENCES}

[1] S. Vazquez, S. Lukic, E. Galvan, L. Franquelo, and J. Carrasco, "Energy storage systems for transport and grid applications," IEEE Trans. Ind. Electron., vol. 57, no. 12, pp. 3881-3895, 2010.
[2] S.-D. Oh, K.-Y. Kim, S.-B. Oh, and H.-Y. Kwak, "Optimal operation of a 1-kw pemfc-based micro-chp system for residential applications," Applied Energy, vol. 96, pp. 93-101, 2012.

[3] W. Schmittinger and A. Vahidi, "A review of the main parameters influencing long-term performance and durability of pem fuel cells," Journal of power sources, vol. 19, pp. 291-312, 2008.

[4] E. Breaz, F. Gao, A. Miraoui, and R. Tirnovan, "A short review of aging mechanism modeling of proton exchange membrane fuel cell in transportation applications," IEEE IECON'14, pp. 3941-3947, 2014.

[5] S. Yin, X. Li, H. Gao, and O. Kaynak, "Data-based techniques focused on modern industry: An overview," IEEE Trans. Ind. Electron., vol. 62, no. 1, pp. 657-667, January 2015.

[6] Z. Li, R. Outbib, S. Giurgea, and D. Hissel, "Diagnosis for pemfc systems: A data- driven approach with the capabilities of online adaptation and novel fault detection," IEEE Trans. Ind. Electron., vol. 62, no. 8, pp. 5164-5174, August 2015.

[7] K. Javed, R. Gouriveau, N. Zerhouni, and P. Nectoux, "Enabling health monitoring approach based on vibration data for accurate prognostics," IEEE Trans. Ind. Electron., vol. 62, no. 1, pp. 647-656, 2015.

[8] X. Si, "An adaptive prognostic approach via nonlinear degradation modelling: Application to battery data," IEEE Trans. Ind. Electron., vol. 62, no. 8, pp. 5082-5096, August 2015.

[9] Y. Xiang and Y. Liu, "Efficient probabilistic methods for real-time fatigue damage prognosis," Annual Conference of the PHM Society, 2010.

[10] X. Zhang and P. Pisu, "An unscented kalman filter based approach for the health monitoring and prognostics of a polymer electrolyte membrane fuel cell," Annual conference of the prognostics and health management, 2012.

[11] M. Jouin, R. Gouriveau, D. Hissel, M. Pera, and N. Zerhouni, "Prognostics of proton exchange membrane fuel cell stacks in a particle filtering framework including characterization disturbances and voltage recovery," IEEE PHM conference, pp. 1-6, 2014.

[12] M. Bressel, M. Hilairet, D. Hissel, and B. O. Bouamama, "Remaining useful life prediction and uncertainty quantification of proton exchange membrane fuel cell under variable load," IEEE Transaction on Industrial Electronics, vol. 63, no. 4, pp. 2569 - 2577, 2016.

[13] ISO, "Iso 13381-1: Condition monitoring and diagnostics of machinery -prognostics - part1: General guidelines," 2004.

[14] M. Orchard, P. Hevia-Kock, B. Zhang, and L. Tang, "Risk measures for particle-filtering-based state-of-charge prognosis in lithium-ion batteries," IEEE Trans. Ind. Electron., vol. 60, no. 11, pp. 5260-5269, 2013.

[15] A. P. Ompusunggu, J.-M. Papy, and S. Vandenplas, "Kalman filtering based prognostics for automatic transmission clutches," IEEE Trans. Mechatron., 2015.

[16] J. Larminie and A. Dicks, Fuel Cell Systems Explained, 2nd ed. John Wiley \& Sons, 2003.

[17] S. Sankararaman and K. Goebel, "Remaining useful life estimation in prognostics: An uncertainty propagation problem," Aerospace Conference 2013, 2013.

[18] P. Lall, R. Lowe, and K. Goebel, "Prognostics health management of electronic systems under mechanical shock and vibration using kalman filter models and metrics," IEEE Trans. Ind. Electron., vol. 59, no. 11, pp. 4301-4314, 2012.

[19] S. Sankararaman, M. J. Daigle, and K. Goebel, "Uncertainty quantification in remaining useful life prediction using first-order reliability methods," IEEE Trans. Rel., vol. 63, no. 2, pp. 603-619, 2014.

[20] K. Yuen, P. Liang, and S. Kuok, "Online estimation of noise parameters for kalman filter," Structural Engineering \& Mechanics, vol. 47, no. 3, 2013. 